\title{
Pattern closure of groups of tree automorphisms
}

\section{Zoran Šunić}

Received: 8 December 2010 / Revised: 27 February 2011 / Accepted: 8 March 2011 / Published online: 24 March 2011

(C) The Author(s) 2011. This article is published with open access at SpringerLink.com

\begin{abstract}
It is shown that every group of automorphisms of a regular rooted tree that is defined by forbidding a set of patterns of size $s+1$ is the topological closure of a self-similar, countable, regular branch group, branching over its level $s$ stabilizer. As an application, it is shown that there are no infinite, finitely constrained, topologically finitely generated groups of binary tree automorphisms defined by forbidden patterns of size two.
\end{abstract}

Keywords Closed self-similar groups · Finitely constrained groups ·

Patterns on trees · Compact groups

Mathematics Subject Classification (2000) $\quad 20 \mathrm{E} 08 \cdot 22 \mathrm{C} 05 \cdot 37 \mathrm{~B} 10$

\section{Introduction}

The group of automorphisms of a regular rooted tree carries three structures, namely a self-similarity structure (related to symbolic dynamics on the tree), a metric structure (with Cantor set topology), and a group theoretic structure (of an iterated wreath product). Each of the tree structures comes with a naturally associated closure operator. Namely, given a set $S$ of tree automorphisms, we may consider the self-similar closure

Communicated by E. Zelmanov.

Partially supported by NSF grant DMS-0805932.

\footnotetext{
Z. Šunić $(\varangle)$

Department of Mathematics, Texas A\&M University,

College Station, TX 77843-3368, USA

e-mail: sunic@math.tamu.edu
} 
of $S$ (the smallest self-similar set containing $S$ ), the topological closure of $S$, and the group closure of $S$ (the group generated by $S$ ). The study of the interaction of these three closures naturally leads to the study of patterns in tree automorphisms.

Informally speaking (see Definition 3 for a precise statement), finitely constrained groups are groups of tree automorphisms defined by forbidding a finite set of patterns (thus they are analogs of shifts of finite type). Note that our notion of a regular branch group (see Definition 2) is a bit wider than the standard one (it reduces to the standard one, as given in [5] or [1], if level transitivity of the action is required). The main reason for this extension is to include the finite, finitely constrained groups into our considerations.

The main results proved here are as follows.

Theorem 1 Let $G$ be a finitely constrained group of tree automorphisms of $X^{*}$ defined by a set of forbidden patterns of size $s+1$, for some $s \geq 0$. Then $G$ is the topological closure (in Aut $\left(X^{*}\right)$ ) of a self-similar, countable, regular branch group $H$, branching over its level s stabilizer.

As an application of Theorem 1, we prove the following.

Theorem 2 There are no infinite, finitely constrained, topologically finitely generated groups of binary tree automorphisms defined by forbidden patterns of size at most 2 .

Note that the closure of the first Grigorchuk group is an infinite, finitely constrained, topologically finitely generated group of binary tree automorphisms defined by patterns of size 4 [6]. The closures of the groups defined by polynomials in [12] provide examples of infinite, finitely constrained, topologically finitely generated groups of binary tree automorphisms defined by patterns of size $s$ (but not size $s-1$ ), for any $s \geq 4$. Thus, by Theorem 2, the question of existence of infinite, finitely constrained, topologically finitely generated groups of binary tree automorphisms defined by patterns of size $s$ remains open only for $s=3$.

The necessary background on groups of automorphisms of rooted regular trees is provided in the next two sections, which are followed by a section in which the main results are proved. More extensive background information may be found in $[1,6,8,11]$.

\section{Background on symbolic dynamics on rooted trees}

\subsection{Rooted trees}

Let $X$ be a finite alphabet of cardinality $k$ (our standard choice is $X=\{0,1, \ldots, k-1\}$ ). The rooted tree $X^{*}$ over $X$ is the rooted, $k$-ary tree in which the vertices are the finite words over $X$, the empty word $\emptyset$ is the root and every vertex $u$ is connected by $k$ directed edges to its $k$ children $u x$, for $x$ in $X$. The edge connecting $u$ to $u x$ is labeled by $x$. Level $n$ of the tree $X^{*}$ is the set $X^{n}$ of words of length $n$ over $X$. We use $X^{*}$ to denote both the rooted tree over $X$ and the set of all words over $X$. Note also that the rooted tree $X^{*}$ is the right Cayley graph of the free monoid $X^{*}$ over $X$. 


\subsection{Portrait space}

Let $A$ be a finite alphabet (in order to avoid confusion, this alphabet is usually disjoint from $X$ ). The portrait space on the tree $X^{*}$ over the alphabet $A$ is the space $A^{X^{*}}$ of all maps from $X^{*}$ to $A$. This space is also called the shift space or the full shift space on $X^{*}$ over the alphabet $A$. The elements of $A^{X^{*}}$ are called portraits ( $X$-tree portraits over $A$ ). For a portrait $g$ in the portrait space, denote by $g_{(u)}$ the symbol from $A$ at vertex $u$ in the tree (note that $(u)$ is in the subscript position with respect to $g$ ). The symbol $g_{(u)}$ is sometimes called the decoration at $u$ in the portrait $g$ and the alphabet $A$ is called the decoration alphabet.

The portrait space $A^{X^{*}}$ is a metric space in which, for distinct portraits $g$ and $h$, the distance is given by

$$
d(g, h)=\sup \left\{\frac{1}{2^{|u|}} \mid u \in X^{*}, g_{(u)} \neq h_{(u)}\right\} .
$$

The topology on $A^{X^{*}}$ is just the product topology on $A^{X^{*}}$ induced by the discrete finite space $A$. Thus, as long as $|A| \geq 2, A^{X^{*}}$ is a Cantor set (in particular, it is compact).

For $u$ in $X^{*}$, the section map $\sigma_{u}: A^{X^{*}} \rightarrow A^{X^{*}}$ at $u$ (also known as the shift map) on the portrait space is defined by

$$
\left(\sigma_{u}(g)\right)_{(v)}=g_{(u v)} .
$$

The section maps provide a right action of $X^{*}$ on the portrait space by continuous maps. Note that, more generally, portrait spaces may be defined over any semigroup (not only over the free monoid $X^{*}$ as defined here; see for instance [4]).

Definition 1 A set of portraits is self-similar if it is invariant under the section maps.

Self-similar sets are also called $X^{*}$-invariant or shift invariant sets.

The case $k=1$ (i.e., $|X|=1$ ) is not excluded from our considerations. In this case the tree $X^{*}$ has the structure of a ray (one-way infinite path), the monoid $X^{*}$ is isomorphic to the monoid of natural numbers $\mathbb{N}$ and the shift space $A^{\mathbb{N}}$ is just the standard one-dimensional one-sided shift (see [10] or [9]).

\subsection{Forbidden patterns}

Let $s \geq 1$. Rooted tree of size $s$ over $X$ is the subtree of $X^{*}$ consisting of the vertices in $X^{[s]}=\cup_{i=0}^{s-1} X^{i}$ (the subtree is also denoted by $X^{[s]}$ ). An $X$-tree pattern of size $s$ over $A$ is a map in $A^{X^{[s]}}$. All $8 X$-tree patterns, where $X=\{0,1\}$, of size 2 over $A=\{\square, \boldsymbol{\square}\}$ are presented in Fig. 1. A tree portrait $g$ contains the tree pattern $p$ of size $s$ at the vertex $u$ if $g_{(u v)}=p_{(v)}$, for $v \in X^{[s]}$.

Let $\mathcal{F}$ be any set of $X$-tree patterns over $A$. Denote by $\mathcal{G}(\mathcal{F})$ the set of all portraits in the portrait space $A^{X^{*}}$ that do not contain any pattern from $\mathcal{F}$ at any vertex. A subset $G$ of the portrait space is defined by a set of forbidden patterns if $G=\mathcal{G}(\mathcal{F})$ for some set of tree patterns $\mathcal{F}$. The set $\mathcal{F}$ is called the set of forbidden tree patterns defining $G$. 

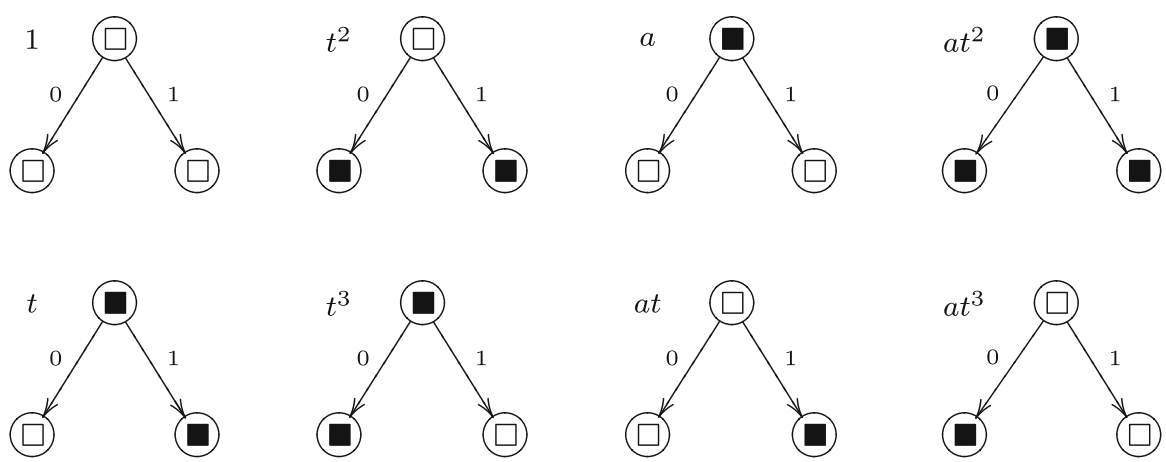

Fig. 1 Patterns of size 2

The following characterization of closed, self-similar sets of portraits may be easily proved in an analogous way as the corresponding claim in case of one-sided shifts.

Theorem 3 Let $G$ be a set of $X$-tree portraits over A. The following are equivalent.

(i) $G$ is closed, self-similar subset of the full tree portrait space $A^{X^{*}}$.

(ii) $G$ is defined by a set of forbidden X-tree patterns.

Closed self-similar sets of portraits are called portrait subspaces (or sometimes portrait spaces, shifts, or subshifts). A portrait space defined by finitely many forbidden patters is called a portrait space of finite type.

Example 1 Let $X=\{0,1\}, A=\{\square, \square\}$ and consider the $X$-tree patterns of size 2 over $A$ provided in Fig. 1.

If we forbid the patterns in the bottom row, i.e., we define the set of forbidden patterns $\mathcal{B}=\left\{t, t^{3}, a t, a t^{3}\right\}$, the automorphisms in the corresponding portrait space of finite type $\mathcal{G}(\mathcal{B})$ can be characterized as follows. A portrait $g$ belongs to $\mathcal{G}(\mathcal{B})$ if and only if, for every vertex $u$ in $X^{*}$,

$$
g_{(u 0)}=g_{(u 1)} \text {. }
$$

Similarly, if we forbid the patterns in the right half of Fig. 1, i.e., we define the set of forbidden patterns $\mathcal{R}=\left\{a, a t^{2}, a t, a t^{3}\right\}$, a portrait $g$ belongs to the portrait space of finite type $\mathcal{G}(\mathcal{R})$ if and only if, for $u$ in $X^{*}$,

$$
g_{(u)}+g_{(u 0)}+g_{(u 1)}=0,
$$

where we interpret the addition and the equality modulo 2, and we interpret $\square$ and as 0 and 1 , respectively.

\section{Background on groups of tree automorphisms}

Let $X^{*}$ be a rooted k-ary tree. We consider the special case when the alphabet $A$ is the finite symmetric group $\mathrm{S}(X)$, i.e., the case when the decoration at each vertex of the 
tree is a permutation of the alphabet $X$. Every portrait $g$ of this type defines a rooted tree automorphism of $X^{*}$, also denoted by $g$, defined by

$$
g\left(x_{1} x_{2} \ldots x_{n}\right)=g_{(\emptyset)}\left(x_{1}\right) g_{\left(x_{1}\right)}\left(x_{2}\right) \ldots g_{\left(x_{1} x_{2} \ldots x_{n-1}\right)}\left(x_{n}\right) .
$$

Conversely, if $g$ is a tree automorphism, it defines a portrait on $X^{*}$, also denoted by $g$, where the permutation of $X$ at the vertex $u$ is uniquely determined by

$$
g_{(u)}(x)=y \Longleftrightarrow g(u x)=g(u) y,
$$

for $x$ and $y$ in $X$.

The group Aut $\left(X^{*}\right)$ of rooted tree automorphisms of $X^{*}$ inherits the self-similarity and the metric structure from the $X$-tree portrait space $\mathrm{S}(X)^{X^{*}}$. In particular, Aut $\left(X^{*}\right)$ is compact and so is each of its closed subgroups.

Note that $\operatorname{Aut}\left(X^{*}\right)$ has the structure of an iterated permutational wreath product

$$
\operatorname{Aut}\left(X^{*}\right) \cong \mathrm{S}(X) \ltimes\left(\operatorname{Aut}\left(X^{*}\right)\right)^{X}=\mathrm{S}(X) \text { < } \operatorname{Aut}\left(X^{*}\right)=\mathrm{S}(X)<(\mathrm{S}(X)<(\mathrm{S}(X) \text { < } \ldots))
$$

where the isomorphism $\operatorname{Aut}\left(X^{*}\right) \cong \mathrm{S}(X) \ltimes\left(\operatorname{Aut}\left(X^{*}\right)\right)^{X}$ is given by

$$
g \mapsto g_{(\emptyset)}\left(\left.g\right|_{0},\left.g\right|_{1}, \ldots,\left.g\right|_{k-1}\right),
$$

and, for $x \in X$, the automorphism $\left.g\right|_{x}$ is just the section $\sigma_{x}(g)$ of $g$ at $x$. The right hand side of (2) is called the decomposition of $g$. If we identify $\operatorname{Aut}\left(X^{*}\right)$ and $\mathrm{S}(X) \ltimes$ $\left(\operatorname{Aut}\left(X^{*}\right)\right)^{X}$ under this isomorphism then, for any two automorphisms $g$ and $h$,

$$
\begin{aligned}
g h & =g_{(\emptyset)}\left(\left.g\right|_{0}, \ldots,\left.g\right|_{k-1}\right) h_{(\emptyset)}\left(\left.h\right|_{0}, \ldots,\left.h\right|_{k-1}\right) \\
& =g_{(\emptyset)} h_{(\emptyset)}\left(\left.g\right|_{h(0)} h_{\mid} 0, \ldots,\left.\left.g\right|_{h(k-1)} h\right|_{k-1}\right) .
\end{aligned}
$$

We will make use of the equalities

$$
\left.(f \cdot g)\right|_{u}=\left.\left.f\right|_{g(u)} \cdot g\right|_{u} \text { and }\left.\left(g^{-1}\right)\right|_{u}=\left(\left.g\right|_{g^{-1}(u)}\right)^{-1}
$$

expressing the sections of products and inverses as products and inverses of appropriate sections.

Definition 2 A self-similar group $H$ is a regular branch group over its level $s$ stabilizer $H_{s}$ if and only if for all $h_{0}, \ldots, h_{k-1} \in H_{s}$ the tree automorphism $\left(h_{0}, h_{1}, \ldots, h_{k-1}\right)$ is also an element of $H_{S}$.

For a set of tree automorphisms $S$ we may define the group $\langle S\rangle$ generated by $S$, the (topological) closure $\bar{S}$ of $S$ and the smallest self-similar set $\tilde{S}$ of tree automorphisms containing $S$ (it consist of all sections of all elements in $S$ ). Further, we can combine these closure operators. For instance, the closure of a group of tree automorphisms is a group and therefore $\overline{\langle S\rangle}$ is the smallest closed group containing $S$. Similarly, the closure of a self-similar set is self-similar and therefore $\overline{\tilde{S}}$ is the smallest closed self-similar 
set containing $S$. Finally, a group of tree automorphisms generated by a self-similar set is self-similar and therefore $\langle\tilde{S}\rangle$ is the smallest self-similar group containing $S$. The smallest closed self-similar group of tree automorphisms containing $S$ is $\overline{\langle\tilde{S}\rangle}$.

A closed group $G$ of tree automorphisms is topologically finitely generated if $G=$ $\overline{\langle S\rangle}$ for some finite set $S$. A tree automorphism $g$ is a finite-state automorphism (we also say that $g$ is defined by a finite automaton) if $\tilde{g}$ (the set of sections of $g$ ) is finite. A group $G$ of tree automorphisms is called an automaton group if it is generated by a finite self-similar set, i.e., $G=\langle\tilde{S}\rangle$, where $\tilde{S}$ is finite. A self-similar group $G$ of tree automorphisms is contracting if there exist a finite set $\mathcal{N}$ of automorphisms such that, for every $g$ in $G$, there exists a level $n$ (depending on $g$ ) such that, for all $m \geq n$, all sections of $g$ at level $m$ are elements of $\mathcal{N}$. Note that finitely generated contracting groups are automaton groups (each element of a contracting group has only finitely many distinct sections, so it is a finite-state automorphism).

The $X$-tree patterns of size $s$ over the alphabet $\mathrm{S}(X)$ act on the finite rooted tree of depth $s$ as in (1), for $n \leq s$. This allows us to identify the set of patterns of size $s$ with the automorphism group Aut $\left(X^{s}\right)$ of the tree of depth $s$. Note that the patterns of size $s$ are defined on $s$ levels ( 0 through $s-1)$, while the tree of depth $s$ has $s+1$ levels (0 through $s$ ).

The following two results are straightforward.

Proposition 1 Let $G=\mathcal{G}(\mathcal{F})$ be a closed, self-similar subset of the tree portrait space $\mathrm{S}(X)^{X^{*}}$ defined by a set of forbidden patterns $\mathcal{F}$. The set $G$ is a subgroup of $\operatorname{Aut}\left(X^{*}\right)$ if and only if, for every $s \geq 1$, the set of essential patterns $\mathcal{E} \mathcal{F}_{s}$ of size $s$ (patterns of size $s$ that actually appear in some element of $G$ ) forms a subgroup of $\operatorname{Aut}\left(X^{s}\right)$ (the automorphism group of the finite regular tree over $X$ of depth $s$ ).

In case $\mathcal{F}$ is a finite set of patterns of size $s, G$ is a group if and only if $\mathcal{E}_{s}$ is a subgroup of $\operatorname{Aut}\left(X^{s}\right)$.

Theorem 4 Let $G$ be a group of tree automorphisms of $X^{*}$. The following are equivalent.

(i) The group $G$ is closed, self-similar subgroup of $\operatorname{Aut}\left(X^{*}\right)$.

(ii) The group $G$ is defined by a set of forbidden patterns.

Definition 3 A group of tree automorphisms defined by a finite set of forbidden patterns is called a finitely constrained group.

A more appropriate term for finitely constrained groups would probably be groups of finite type, as in [6], where this kind of groups were introduced, but this term seems to be already overused and so we will avoid it (the term finitely constrained group was used for the first time in [7]).

Example 2 Consider again the patterns of size 2 given in Fig. 1 and interpret $\square$ as the trivial permutation of $X=\{0,1\}$ and $\mathbf{a}$ as the non-trivial permutation (01). The group Aut $\left(X^{2}\right)$ of tree automorphisms of the $X$-tree of depth 2 is isomorphic to the dihedral group $D_{4}$ and is generated by the automorphism $t$ of order 4 and the automorphism $a$ of order 2 (subject to the relation $a t a=t^{3}$ ). 
Note that, for self-similar groups of binary tree automorphisms, being infinite and being transitive on each level of the tree are equivalent properties (see [2] or [3, Lemma 3, p. 112]).

The only proper transitive subgroups of $\operatorname{Aut}\left(X^{2}\right)$ are the groups $\left\{1, t, t^{2}, t^{3}\right\}$, with complement $\mathcal{R}$, and the group $\left\{1, a, t^{2}, a t^{2}\right\}$, with complement $\mathcal{B}$. Thus, $\mathcal{G}(\mathcal{R})$ and $\mathcal{G}(\mathcal{B})$ are the only infinite, finitely constrained groups of binary tree automorphisms defined by forbidden patterns of size 2 (in addition to the full group $\operatorname{Aut}\left(X^{*}\right)$, which is defined by declaring the empty set to be the set of forbidden patterns). The group $\mathcal{G}(\mathcal{B})$ appears explicitly in [6, p. 174] as a simple example of a finitely constrained group that is neither finite nor equal to $\operatorname{Aut}\left(X^{*}\right)$.

To complete our understanding of all groups defined by patterns of size 2 , note that the only other subgroups of $\operatorname{Aut}\left(X^{2}\right)$ that define nontrivial finitely constrained groups are $\langle a\rangle$ and $\left\langle a t^{2}\right\rangle$. In each case, the corresponding finitely constrained group is cyclic of order 2 .

\subsection{Pattern closure construction}

Given a self-similar group $K$ and we may construct the finitely constrained group $\mathcal{G}\left(\mathcal{F}_{s}(K)\right)$ defined by the set of forbidden patterns $\mathcal{F}_{S}(K)$ of size $s$, which is simply the set of patterns of size $s$ that do not appear in any element of $K$.

We note that the following corollary of Theorem 1 shows that the contraction property is, in a sense, compatible with the pattern closure construction.

Corollary 1 Let $G$ be a finitely constrained group of tree automorphisms of $X^{*}$ defined by allowing all patterns of size $s+1$, for some $s \geq 0$, that appear in some self-similar, contracting group $K$ (and forbidding those that do not). Then $G$ is the topological closure (in Aut $\left(X^{*}\right)$ ) of a self-similar, contracting, countable, regular branch group $H$, branching over its level s stabilizer $H_{S}$.

Theorem 1 may be seen as a refinement of the direction (ii) implies (i) of Theorem 5 below, since $H$ can be chosen to be countable and the proof provides an explicit way to construct it.

Theorem 5 Let $G$ be a group of tree automorphisms of $X^{*}$ and $s \geq 0$. The following are equivalent.

(i) The group $G$ is the closure of some self-similar, regular branch group $H$, branching over its level s stabilizer $H_{s}$.

(ii) The group $G$ is finitely constrained group defined by patterns of size $s+1$.

The direction (ii) implies (i) is proved in [6, Proposition 7.5] and the other direction in [12, Theorem 3].

The following example of the pattern closure construction plays a role in the proof of Theorem 2.

Example 3 The group $\mathcal{G}(\mathcal{R})$ from Example 1 is just one example in the family of finitely constrained groups defined by the pattern closure construction with respect to 
various sizes applied to the, so called, odometer group (the group $\mathcal{G}(\mathcal{R})$ corresponds to size 2).

The $k$-ary odometer automorphism $t$ of $X^{*}$ is defined by

$$
t=\rho(1,1, \ldots, 1, t)
$$

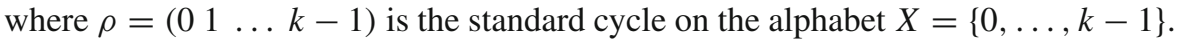
The group $T=\langle t\rangle$ is self-similar, contracting, level transitive group.

For a fixed size $s+1, s \geq 0$, define $G(k, s+1)=\mathcal{G}\left(\mathcal{F}_{s+1}(T)\right)$ as the finitely constrained group of $k$-ary rooted tree automorphisms for which the forbidden patterns are precisely the patterns of size $s+1$ that do not appear in any element of $\langle t\rangle=T \cong \mathbb{Z}$.

\section{Proofs of Theorem 1 and Theorem 2 (and Corollary 1)}

For a word $u$ over $X$ and a tree automorphism $f$, denote by $\delta_{u}(f)$ the unique tree automorphism that stabilizes level $|u|$ and has trivial section at each vertex at level $|u|$ except at $u$ where its section is equal to $f$.

Lemma 1 Let $h$ and $g$ be automorphisms of the tree $X^{*}$. For any vertex $u$,

$$
\left(\delta_{u}(h)\right)^{g}=\delta_{v}\left(h^{\left.g\right|_{v}}\right),
$$

where $v=g^{-1}(u)$.

Proof Let $|u|=n$ and $v$ be arbitrary vertex at level $n$. Since $\delta_{u}(h)$ stabilizes level $n$ of $X^{*}$, we have $\left(\delta_{u}(h)\right)^{g}=g^{-1} \delta_{u}(h) g(v)=g^{-1} g(v)=v$. Thus $\left(\delta_{u}(h)\right)^{g}$ stabilizes level $n$. Further,

$$
\begin{aligned}
\left.\delta_{u}(h)\right)\left.^{g}\right|_{v} & =\left.\left(g^{-1} \delta_{u}(h) g\right)\right|_{v}=\left.\left.\left.g^{-1}\right|_{\delta_{u}(h) g(v)} \cdot \delta_{u}(h)\right|_{g(v)} \cdot g\right|_{v} \\
& =\left.\left.\left.g^{-1}\right|_{g(v)} \cdot \delta_{u}(h)\right|_{g(v)} \cdot g\right|_{v}=\left.\left.\left(\left.g\right|_{g^{-1} g(v)}\right)^{-1} \cdot \delta_{u}(h)\right|_{g(v)} \cdot g\right|_{v} \\
& =\left.\left.\left(\left.g\right|_{v}\right)^{-1} \delta_{u}(h)\right|_{g(v)} g\right|_{v}=\left(\left.\delta_{u}(h)\right|_{g(v)}\right)^{\left.g\right|_{v}} \\
& = \begin{cases}h^{\left.g\right|_{v}}, & g(v)=u \\
1, & g(v) \neq u,\end{cases}
\end{aligned}
$$

showing that $\left(\delta_{u}(h)\right)^{g}=\delta_{v}\left(h^{\left.g\right|_{v}}\right)$, where $v=g^{-1}(u)$.

Proof of Theorem 1 Let $\mathcal{P}$ be the set of patterns of size $s+1$ appearing in the elements of the self-similar group $G$. Since $G$ is self-similar, this is the set of patterns of size $s+1$ appearing at the root in the elements of $G$. Let $S=\left\{g_{1}, \ldots, g_{m}\right\}$ be a set of elements in $G$ such that every pattern in $\mathcal{P}$ appears at the root in at least one of the automorphisms in $S$ (note that the set $\mathcal{P}$ is finite, so $S$ may be chosen to be finite as well). Let $L=\langle\tilde{S}\rangle$ be the smallest self-similar group containing $S$ (this is a subgroup of $G$ ) and let $L_{s}=\left\langle S^{\prime}\right\rangle$ be the stabilizer of level $s$ in $L$. Note that $\tilde{S}$ is countable (since $S$ is finite and every tree automorphism has no more than countably many sections). Therefore $L$ is countable and so are $L_{s}$ and $S^{\prime}$. 
Let

$$
D=\left\{\delta_{u}(h) \mid h \in S^{\prime}, u \in X^{*}\right\}
$$

and

$$
H=\langle D \cup \tilde{S}\rangle
$$

Note that $H$ is self-similar. Indeed, $H=\langle D \cup L\rangle$ and all sections of the elements in $D \cup L$ are trivial or elements in the self-similar group $L$. Therefore $D \cup L$ is a self-similar set and $H$ itself is self-similar.

We claim that $H_{S}=\langle D\rangle$.

Since every element $h \in S^{\prime}$ stabilizes the first $s$ levels of the tree $X^{*}, \delta_{u}(h)$ stabilizes $s+|u|$ levels. Therefore $\langle D\rangle$ is a subgroup of $H_{S}$.

Further, by Lemma 1, for any word $u, g \in \tilde{S}$ and $h \in S^{\prime},\left(\delta_{u}(h)\right)^{g}=\delta_{v}\left(h^{\left.g\right|_{v}}\right)$, where $v=g^{-1}(u)$. Since $L_{s}$ is normal in $L$, there exist $h_{1}, \ldots, h_{r} \in S^{\prime}$ and exponents $\epsilon_{1}, \ldots, \epsilon_{r}$ in $\{-1,1\}$ such that $h^{\left.g\right|_{v}}=h_{1}^{\epsilon_{1}} \ldots h_{r}^{\epsilon_{r}}$. Therefore

$$
\left(\delta_{u}(h)\right)^{g}=\delta_{v}\left(h^{\left.g\right|_{v}}\right)=\delta_{v}\left(h_{1}^{\epsilon_{1}} \ldots h_{r}^{\epsilon_{r}}\right)=\delta_{v}\left(h_{1}\right)^{\epsilon_{1}} \ldots \delta_{v}\left(h_{r}\right)^{\epsilon_{r}} .
$$

The last equality shows that the group $\langle D\rangle$ is normal subgroup of $H$.

Since $\langle D\rangle$ is normal in $H=\langle D \cup \tilde{S}\rangle$, any element of $H_{S}$ can be written as a product of an element in $\langle D\rangle$ and an element in $\langle\tilde{S}\rangle=L$ stabilizing $s$. But the generators of $L_{s}$ are in $\langle D\rangle$, which shows that $H_{S}=\langle D\rangle$.

The group $H$ is a regular branch group, branching over its stabilizer $H_{s}$ of level $s$. This is clear since, for any words $u_{0}, \ldots, u_{k-1}$ in $X^{*}$, and elements $h_{0}, \ldots, h_{k-1}$ in $S^{\prime}$,

$$
\begin{aligned}
\left(\delta_{u_{0}}\left(h_{0}\right), \ldots, \delta_{u_{k-1}}\left(h_{k-1}\right)\right) & =\left(\delta_{u_{0}}\left(h_{0}\right), 1, \ldots, 1\right) \cdots\left(1, \ldots, \delta_{u_{k-1}}\left(h_{k-1}\right)\right) \\
& =\delta_{0 u_{0}}\left(h_{0}\right) \cdots \delta_{(k-1) u_{k-1}}\left(h_{k-1}\right) \in H_{s} .
\end{aligned}
$$

By Theorem 5, the closure $\bar{H}$ is a finitely constrained group, defined by patterns of size $s+1$. Moreover, since $H$ is self-similar, the patterns defining $\bar{H}$ are the patterns of size $s+1$ appearing at the root in the elements of $H$. Since, for nonempty words $u$ and $h \in S^{\prime}, \delta_{u}(h)$ stabilizes level $s+1$, the patterns of size $s+1$ appearing at the root in the elements of $H$ are precisely the patterns of size $s+1$ appearing at the root of the elements in $L$, and these are the patterns defining $G$. Therefore $\bar{H}=G$.

Proof of Corollary 1 Assume that $K$ is contracting over the finite set $\mathcal{N}$.

Let $\mathcal{P}$ be the set of patterns of size $s+1$ appearing in the elements of $K$. Let $S=\left\{g_{1}, \ldots, g_{m}\right\}$ be a set of elements in $K$ such that $S$ contains $\mathcal{N}$ and every pattern in $\mathcal{P}$ appears at the root in at least one of the automorphisms in $S$. From this point on the construction of $H$ follows the same steps as the one in the proof of Theorem 1.

Since $S$ is finite and $K$ is contracting, $\tilde{S}$ is finite as well. Thus $L$ is an automaton group that is contracting over $\mathcal{N}$. The group $H$ is also contracting over $\mathcal{N}$ since, for 
$h \in S^{\prime}$ each section of $\delta_{u}(h)$ at level $|u|$ is an element of $L$ and $L$ is contracting over $\mathcal{N}$.

Note that, in the contracting case, since $\tilde{S}$ is finite and $L=\langle\tilde{S}\rangle$ is finitely generated, so is its finite index subgroup $L_{S}$. This means that $S^{\prime}$ may be chosen to be finite as well. Further, in some situations, the set $D=\left\{\delta_{u}(h) \mid h \in S^{\prime}, u \in X^{*}\right\}$ in the definition of $H$ may be replaced by some subset such as, for instance, $D^{\prime}=\left\{\delta_{0^{n}}(h) \mid h \in S^{\prime}, n=\right.$ $0,1, \ldots\}$.

The claim of Theorem 2 follows if we prove than none of the groups $\operatorname{Aut}\left(X^{*}\right), \mathcal{G}(\mathcal{B})$, and $\mathcal{G}(\mathcal{R})$ is topologically finitely generated. This is known for $\operatorname{Aut}\left(X^{*}\right)$ (see [6]), the claim for $\mathcal{G}(\mathcal{R})$ is proved in more general form in Proposition 2, and the claim for $\mathcal{G}(\mathcal{B})$ is proved in Proposition 3.

Proposition 2 The finitely constrained group $G(k, s+1)$ (defined in Example 3 by allowing the patterns of size $s+1$ that appear in the odometer group) is not topologically finitely generated, for $k \geq 2, s \geq 0$.

Proof We first explicitly determine a self-similar, countable, regular branch group $H$, branching over its level stabilizer $H_{S}$, such that $G$ is the closure of $H$ in $\operatorname{Aut}\left(X^{*}\right)$. In order to accomplish this we follow the argument in the proof of Theorem 1 (we follow the argument somewhat loosely, since in the concrete situation some obvious simplifications are available).

The role of $L$ may be played by $T$ itself. The stabilizer $T_{S}$ of level $s$ in $T$ is generated by $t^{k^{s}}$. Define $t_{s}=t^{k^{s}}$ and, for $n \geq s$,

$$
t_{n+1}=\left(1,1, \ldots, t_{n}\right)
$$

Let $H=\left\langle t, t_{s+1}, \ldots\right\rangle$. Then, for the level $s$ stabilizer in $H$, we have

$$
H_{s}=\left\langle t_{n}^{t^{i}} \mid n \geq s, i=0, \ldots, k^{n-s}-1\right\rangle
$$

The closure $\bar{H}$ of $H$ in $\operatorname{Aut}\left(X^{*}\right)$ is precisely $G$. This implies that $G / G_{n}=H / H_{n}$, for $n \geq 0$.

Therefore, in order to show that $G$ is not topologically finitely generated, it is sufficient to show that, for $n \geq s+1$, the minimal number of generators of $H_{[n]}=H / H_{n}$ is $n-s$.

For $n \geq s+1$, let $A_{n}=C_{k^{s+1}} \times C_{k} \times \cdots \times C_{k}$, where $C_{m}$ denotes the standard cyclic group of order $m$ (the elements are the residue classes modulo $m$ ) and the total number of factors is $n-s$. We claim that, for $n \geq s+1$, there exists a surjective homomorphism from $H_{[n]}$ to $A_{n}$.

First, since the generators $t_{n}, t_{n+1}, \ldots$ stabilize level $n$, the group $H_{[n]}$ is generated by (the cosets of) $\left\{t, t_{s+1}, \ldots, t_{n-1}\right\}$. Define a map $\beta_{n}$ from the set of group words over $\left\{t, t_{s+1}, \ldots, t_{n-1}\right\}$ to $A_{n}$ by setting

$$
\beta_{n}(W)=\left(\operatorname{expn}_{t}(W), \operatorname{expn}_{t_{s+1}}(W), \ldots, \operatorname{expn}_{t_{n-1}}(W)\right),
$$

where $\operatorname{expn}_{t_{*}}(W)$ denotes the exponent of the letter $t_{*}$ in $W$. We claim that the map $\beta_{n}$ represents a surjective homomorphism from $H_{[n]}$ to $A_{n}$. The surjectivity and the 
homomorphism property follow trivially, once we show that $\beta_{n}$ is well defined (as a map from $H_{[n]}$ ). Therefore, we need to show that, for every group word $W$ over $\left\{t, t_{s+1}, \ldots, t_{n-1}\right\}$ representing the identity in $H_{[n]}$ (i.e., every group word $W$ representing an element in the stabilizer $\left.H_{n}\right), \beta_{n}(W)=(0,0, \ldots, 0)$. We do this by induction on $n$.

For $n=s+1, H_{[s+1]}=\langle t\rangle$ and, since the smallest power of $t$ stabilizing level $s+1$ is $t^{k^{s+1}}$, any group word over $\{t\}$ representing the identity in $H_{[s+1]}$ is a power of $t^{k^{s+1}}$.

Let $n>s+1$ and assume that the inductive claim is true for $n-1$. Let $W$ be a group word over $\left\{t, t_{s+1}, \ldots, t_{n-1}\right\}$ representing the identity in $H_{[n]}$. In particular, the word $W$ must represent an element of the level stabilizer $H_{s+1}$. Since all generators $t_{s+1}, t_{s+2}, \ldots$ stabilize level $s+1$, we conclude that $\operatorname{expn}_{t}(W)$ must be divisible by $k^{s+1}$. Let $W_{0}, W_{1}, \ldots, W_{k-1}$ be the group words over $\left\{t, t_{s+1}, \ldots, t_{n-2}\right\}$ obtained by decomposition from the word $W$ (see (2) and (3)). Since $W$ represents the identity in $H_{[n]}$ (i.e., it stabilizes level $n$ ), the words $W_{i}, i=0, \ldots, k-1$, represent the identity in $H_{[n-1]}$ (i.e., they stabilize level $\left.n-1\right)$. We have

$$
\begin{gathered}
\operatorname{expn}_{t}\left(W_{0}\right)+\cdots+\operatorname{expn}_{t}\left(W_{k-1}\right)=\operatorname{expn}_{t}(W)+k^{s} \operatorname{expn}_{t_{s+1}}(W), \\
\operatorname{expn}_{t_{s+1}}\left(W_{0}\right)+\cdots+\operatorname{expn}_{t_{s+1}}\left(W_{k-1}\right)=\operatorname{expn}_{t_{s+2}}(W) \\
\cdots \\
\operatorname{expn}_{t_{n-2}}\left(W_{0}\right)+\cdots+\operatorname{expn}_{t_{n-2}}\left(W_{k-1}\right)=\operatorname{expn}_{t_{n-1}}(W)
\end{gathered}
$$

By the induction hypothesis, for $i=0, \ldots, k-1, \operatorname{expn}_{t}\left(W_{i}\right)$ is divisible by $k^{s+1}$, while $\operatorname{expn}_{t_{j}}\left(W_{i}\right)$ is divisible by $k$, for $j=s+1, \ldots, n-2$. Since expn ${ }_{t}(W)$ is also divisible by $k^{s+1}$ we conclude from the first equality in (4) that $\operatorname{expn}_{t_{s+1}}(W)$ is divisible by $k$. The other equalities in (4) imply that $\operatorname{expn}_{t_{s+2}}(W), \ldots, \operatorname{expn}_{t_{n-1}}(W)$ are divisible by $k$.

Since, for $n \geq s+1$, the abelian group $A_{n}$ has rank $n-s$ and $\beta_{n}: H_{[n]} \rightarrow A_{n}$ is a surjective homomorphism, we conclude that the closure $\bar{H}=G$ is not topologically finitely generated.

Proposition 3 The finitely constrained group $G(\mathcal{B})$ (defined in Example 1) is not topologically finitely generated.

Proof The proof follows the general outline of the Proof of Proposition 2.

The role of a self-similar, countable, regular branch group $H$, branching over its first level stabilizer $H_{1}$, such that $G=\mathcal{G}(\mathcal{B})$ is the closure of $H \operatorname{in} \operatorname{Aut}\left(X^{*}\right)$ is played by $H=\left\langle a, a_{1}, a_{2}, a_{3}, \ldots\right\rangle$ and the role of $L$ by $\left\langle a, a_{1}\right\rangle$, where

$$
a=(01)(1,1), \quad a_{1}=(a, a)
$$

and for $n \geq 2$,

$$
a_{n+1}=\left(1, a_{n}\right) \text {. }
$$

Every generator of $H$ has order 2 . 
The group $H_{[n]}=H / H_{n}$, for $n \geq 1$, is generated by (the cosets of) $\left\{a, a_{1}\right.$, $\left.a_{2}, \ldots, a_{n-1}\right\}$. The map $\beta_{n}: H_{[n]} \rightarrow A_{n}$, where $A_{n}=C_{2}^{n}$, defined by

$$
\beta_{n}(W)=\left(\operatorname{expn}_{a}(W), \operatorname{expn}_{a_{1}}(W), \ldots, \operatorname{expn}_{a_{n-1}}(W)\right)
$$

is a surjective homomorphism, which shows that $\bar{H}=\mathcal{G}(\mathcal{B})$ is not topologically finitely generated.

Indeed, to show that $\beta_{n}$, for $n \geq 1$, is a surjective homomorphism it suffices to show that it is well defined, i.e., it suffices to show that for a group word $W$ over $\left\{a, a_{1}, \ldots, a_{n-1}\right\}$ representing an element in $H_{n}$ the exponent $\operatorname{expn}_{a}(W)$ and the exponents $\operatorname{expn}_{a_{i}}(W), i=1, \ldots, n-1$, are even. This can be accomplished by induction on $n$. The claim is clear for $n=1$, since $a$ is the only generator that does not stabilize level 1. In fact, $\exp _{a}(W)$ must be even for any group word over $\left\{a, a_{1}, a_{2}, \ldots\right\}$ stabilizing at least one level of the tree. Assume that $n \geq 2$ and the claim is correct for $n-1$. For any group word $W$ over $a, a_{1}, \ldots, a_{n-1}$ representing an element in $H_{n}$, let the words $W_{0}$ and $W_{1}$ be the group words over $\left\{a, a_{1}, \ldots, a_{n-1}\right\}$ obtained by decomposition. These words represent elements in $H_{n-1}$ and the induction hypothesis applies. Since

$$
\begin{aligned}
\operatorname{expn}_{a}\left(W_{0}\right) & =\operatorname{expn}_{a_{1}}(W), \\
\operatorname{expn}_{a_{1}}\left(W_{0}\right)+\operatorname{expn}_{a_{1}}\left(W_{1}\right) & =\operatorname{expn}_{a_{2}}(W), \\
\cdots & \\
\operatorname{expn}_{a_{n-2}}\left(W_{0}\right)+\operatorname{expn}_{a_{n-2}}\left(W_{1}\right) & =\operatorname{expn}_{a_{n-1}}(W) .
\end{aligned}
$$

and all exponents on the left are even, all exponent on the right are even as well, completing the proof.

Acknowledgments The author would like to thank Rostislav Grigorchuk for stimulating conversations and the referee for useful remarks.

Open Access This article is distributed under the terms of the Creative Commons Attribution License which permits any use, distribution and reproduction in any medium, provided the original author(s) and source are credited.

\section{References}

1. Bartholdi, L., Grigorchuk, R.I., Šunik, Z.: Branch groups. In: Handbook of Algebra, vol. 3, pp. 9891112. North-Holland, Amsterdam (2003). doi:10.1016/S1570-7954(03)80078-5

2. Bondarenko, I., Grigorchuk, R., Kravchenko, R., Muntyan, Y., Nekrashevych, V., Savchuk, D., Šunić, Z.: Groups generated by 3-state automata over a 2-letter alphabet. I. São Paulo J. Math. Sci. 1(1), 1-39 (2007)

3. Bondarenko, I., Grigorchuk, R., Kravchenko, R., Muntyan, Y., Nekrashevych, V., Savchuk, D., Šnić, Z.: On classification of groups generated by 3-state automata over a 2-letter alphabet. Algebra Discrete Math. 1, 1-163 (2008)

4. Coornaert, M., Papadopoulos, A.: Symbolic dynamics and hyperbolic groups. In: Lecture Notes in Mathematics, vol. 1539. Springer, Berlin (1993)

5. Grigorchuk, R.: Just infinite branch groups. In: du Sautoy, M.P.F., Segal, D., Shalev, A. (eds.) New Horizons in Pro- $p$ Groups, pp. 121-179. Birkhäuser, Boston (2000) 
6. Grigorchuk, R.: Solved and unsolved problems around one group. In: Infinite Groups: Geometric, Combinatorial and Dynamical Aspects. Progr. Math., vol. 248, pp. 117-218. Birkhäuser, Basel (2005)

7. Grigorchuk, R., Nekrashevych, V., Šunić, Z.: Hanoi towers group on 3 pegs and its pro-finite closure. Oberwolfach Rep. 25, 15-17 (2006)

8. Grigorchuk, R., Šunić, Z.: Self-similarity and branching in group theory. In: Groups St. Andrews 2005, vol. 1. London Math. Soc. Lecture Note Ser., vol. 339, pp. 36-95. Cambridge University Press, Cambridge (2007). doi:10.1017/CBO9780511721212.003

9. Kitchens, B.P.: Symbolic Dynamics. One-Sided, Two-Sided and Countable State Markov Shifts. Universitext. Springer, Berlin (1998)

10. Lind, D., Marcus, B.: An Introduction to Symbolic Dynamics and Coding. Cambridge University Press, Cambridge (1995). doi:10.1017/CBO9780511626302

11. Nekrashevych, V.: Self-similar groups. In: Mathematical Surveys and Monographs, vol. 117. American Mathematical Society, Providence (2005)

12. Šunić, Z.: Hausdorff dimension in a family of self-similar groups. Geom. Dedicata 124, 213-236 (2007). doi:10.1007/s10711-006-9106-8 